\title{
Acknowledgement of Reviewers for 2020
}

Published online: 11 February 2021

(C) The Author(s), under exclusive licence to Springer Nature B.V. part of Springer Nature 2021

We thank all those scientists and experts in the various fields represented in Surveys in Geophysics for devoting their time and effort to review the papers that we have sent them. The Editor in Chief, Dr. Michael J. Rycroft, and the Publisher acknowledge the colleagues listed below for their most helpful reviews of papers which have been provided during the period January 2020 to December 2020.

Tariq Alkhalifah

Michael Angove

Raphael Antoine

Karen Aplin

Michael Asten

Ali Atef

Jing $\mathrm{Ba}$

Kiyoshi Baba

Anna Belehaki

Trent Biggs

N. F. Blagoveshchenskaya

Guy Brasseur

James Bremer

Jan Brockmann

Natalia Bushenkova

Jianchao Cai

Francisco Calafat

Carlos Castillo

Alan Chave

Shuangquan Chen

Yangkang Chen

Yun-Jhih Chen

Sten Claessens

Andrew Coates

Enrique Cordaro

Julia Correa

Alexander Costall

Caitano da Silva

Lidong Dai

Stephen Darby
José Darrozes
Mihai Datcu
Angelo De Santis
Heiner Denker
Henryk Dobslaw
Guillaume Dodet
Ramin Dokht
John Douglas
Maria Grazia D'Urso
Erle Ellis
Colin Farquharson
Will Featherstone
Xuliang Feng
Fouad Fethi
Horacio Florez
René Forsberg
Martin Fullekrug
Pauline Galea
Antonio Genova
Sia Ghelichkhan
Nat Gopalswamy
Marloes Gutenstein
Alfred Hanssen
Christian Hirt
Steven Hosford
Liangbo Hu
Chao-Song Huang
Michael Hudec

Dryver Huston

Alik Ismail-Zadeh

David Ivers

Martin Jutzeler

Laura Kong

Nikos Koutsias

Chengling Kuo

Chung-Yen Kuo

Yaoguo Li

Desheng Liu

Guochang Liu

Jeffrey Love

Xiao Dong Luo

Alejandro Luque

Jian Wei Ma

Robbie Mackenzie

Oleg Makarynskyy

Subhashis Mallick

Lisa McNeill

Niccolo Menegoni

Antonio Menghini

Sabrina Metzger

David Modesto

Toru Mogi

Mostafa Mousavi

Shane Mule

Hoang Nguyen

Keri Nicoll

Yasuo Ogawa 


$\begin{array}{lll}\text { Patricia Oliva } & \text { Reiner Rummel } & \text { Robert Tenzer } \\ \text { Martin Ostoja-Starzewski } & \text { Juan Santos } & \text { David Themens } \\ \text { Yuichi Otsuka } & \text { Guy Schumann } & \text { Liam Toney } \\ \text { Roland Pail } & \text { Josef Sebera } & \text { Fabio Tosti } \\ \text { Oya Pamukcu } & \text { Roland Seferian } & \text { Ralf Trapp } \\ \text { Xinpeng Pan } & \text { Kurt Seitz } & \text { Andreas Tzanis } \\ \text { Isaak Parcharidis } & \text { Lee Slater } & \text { Ilya Usoskin } \\ \text { Jared Peacock } & \text { Viacheslav Spichak } & \text { David Wiese } \\ \text { Kristof Petrovay } & \text { D. Sarah Stamps } & \text { Artur Wolak } \\ \text { Pilar Queralt } & \text { Anatoly Streltsov } & \text { Xiaozhi Yang } \\ \text { Jean-Pierre Raulin } & \text { Junzhe Sun } & \text { Huajian Yao } \\ \text { Mirko Reguzzoni } & \text { Yang Yi Sun } & \text { Jinhai Zhang } \\ \text { Emiliano Renzi } & \text { Costas Synolakis } & \text { Pei-Zhen Zhang } \\ \text { Aleksandar Ristic } & \text { Akihiro Takeuchi } & \text { Zhaoyun Zong }\end{array}$

We apologize for any errors or inadvertent omissions.

Publisher's Note Springer Nature remains neutral with regard to jurisdictional claims in published maps and institutional affiliations. 